\title{
Opening Base Wedge Osteotomy of the First Metatarsal Using the Arthrex Low Profile Plate and Screw System ${ }^{\text {TM }}$
}

\author{
by Mark A. Hardy, DPM, FACFAS ${ }^{1 凹}$, Jason R. Grove, DPM ${ }^{2}$
}

The Foot and Ankle Online Journal 2 (4): 2

A number of procedures have been described for the high-end hallux valgus deformity. The authors describe a modification of the proximal opening wedge osteotomy utilizing the Arthrex Low Profile Plate and Screw System $^{\mathrm{TM}}$. The system allows for stable fixation of the osteotomy without the need of a bone graft to maintain position. It is hoped that this article will generate a renewed interest in this highly effective procedure.

Key words: Hallux abductovalgus deformity, opening wedge osteotomy, first metatarsal

Published: April, 2009

$\mathrm{F}$ irst metatatarsal base osteotomies have been performed for many years to correct moderate to severe intermetatarsal angles of the hallux abducto valgus deformity. A plethora of procedures have been described. Some of the most common include the crescentic, closing base wedge osteotomy and the chevron osteotomy. In contrast, the opening base wedge osteotomy has not been commonly used among surgeons. Most authors have stated that the opening base wedge is more technically demanding, requiring longer healing time due to the use of a graft, and can lead to joint jamming at the metatarsophalangeal joint.

\footnotetext{
Address correspondence to: Mark A. Hardy, DPM, FACFAS Kaiser Permanente Foundation Department of Podiatric Surgery 12301 Snow Road Parma, OH 44130

E-mail: Markhardy@sbcglobal.net.

${ }^{1}$ Director, Foot \& Ankle Trauma Service, Kaiser Permanente, Cleveland, Ohio. Cleveland Clinic Foundation Residency Program.

${ }^{2}$ Second-year resident (PGY-2), Kaiser Permanente/Cleveland Clinic Foundation Residency Program, Cleveland, Ohio.
}

Arthrex, Inc. has introduced a low profile titanium plate with a central spacer to allow accurate and more stable correction of the opening base wedge osteotomy. (Figs. 1,2) Furthermore, the use of the Arthrex Low Profile Plate and Screw System ${ }^{\text {TM }}$ eliminates the need for a bone graft to hold the metatarsal in the corrected position. This makes the procedure less cumbersome than it may have been in the past.

\section{Surgical technique}

The incision should begin just proximal to the first metatarsocuneiform joint and end at the base of the proximal phalanx of the hallux. (Fig. 3) A modified McBride procedure is performed and the medial eminence is harvested for the future bone graft. The base of the first metatarsal is then exposed both medially and dorsally. 


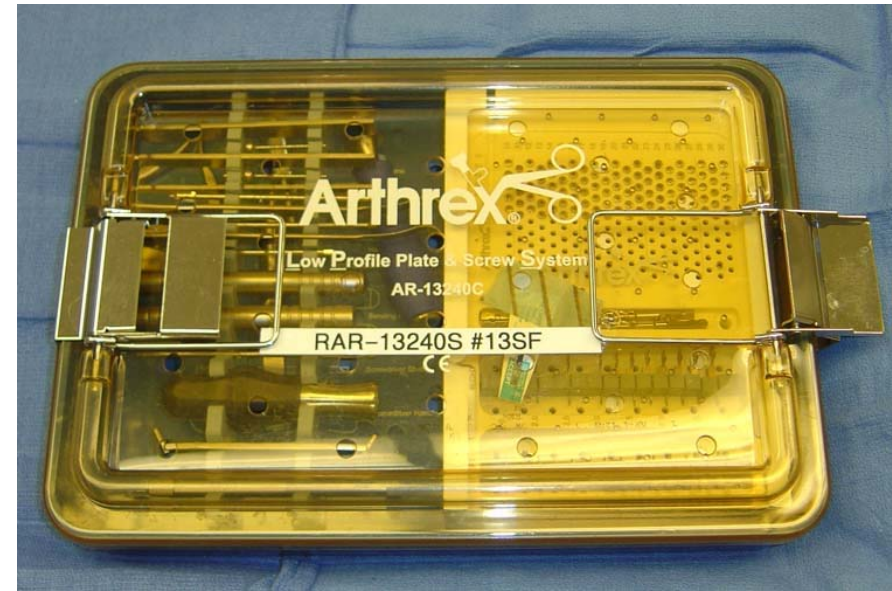

Figure 1 The Arthrex Low Profile Plate and Screw System $^{\mathrm{TM}}$.

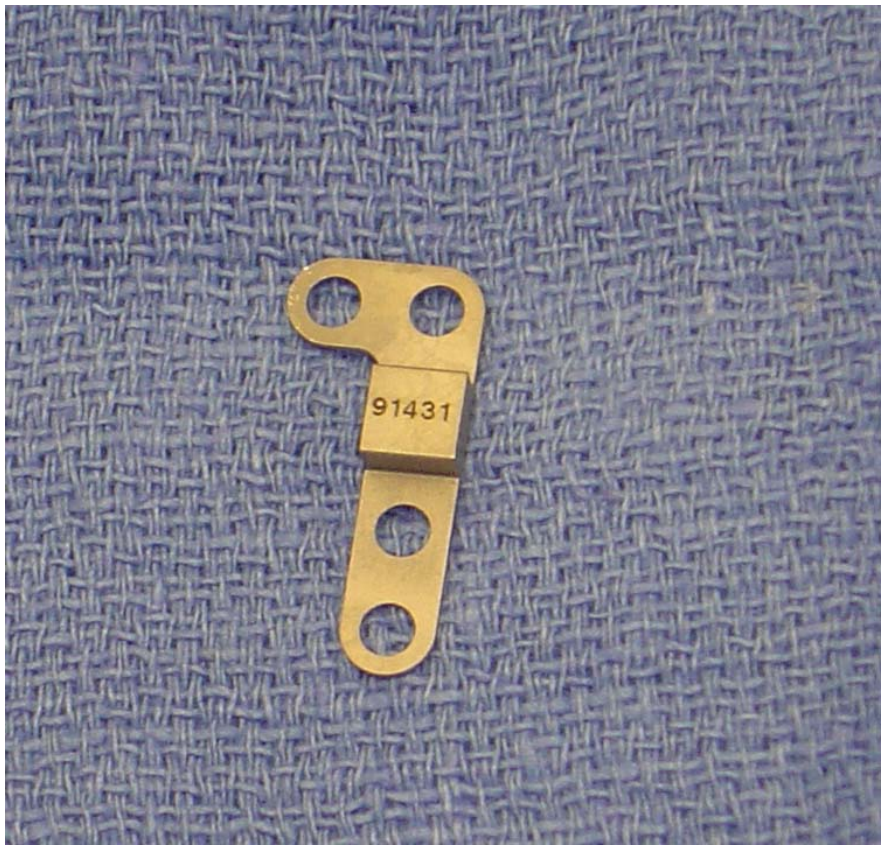

Figure 2 The opening wedge plate.

The first metatarsocuneiform joint is identified and a skin scribe is used to mark the dorsolateral cortex $1 \mathrm{~cm}$ distal to the metatarsocuneiform joint. (Fig. 4) A 0.045 inch Kirschner wire is then driven from dorsal to plantar at the site already marked. The wire is driven perpendicular to the weight bearing surface to serve as an axis guide as is similarly done when performing a closing base wedge osteotomy. (Fig. 5 and 6)

(C) The Foot and Ankle Online Journal, 2009

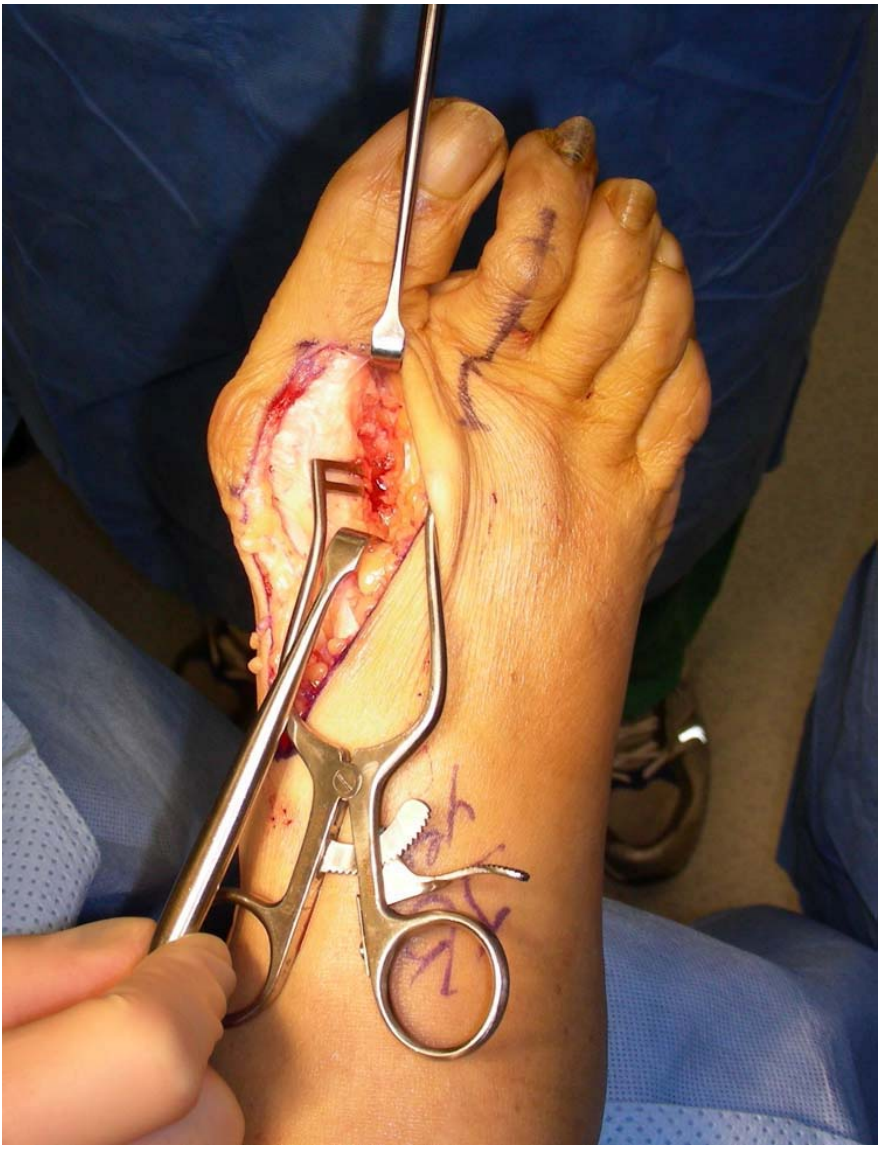

Figure 3 The standard incision allowing for interspace dissection and access to performing the opening base wedge osteotomy.

The technique guide recommends performing the osteotomy halfway between and perpendicular to the metatarsal and the weight bearing surface; we prefer to make this cut entirely perpendicular to the weight bearing surface, as we feel this effects less sagittal plane elevation.

Once the k-wire is in the proper position, a sagittal saw is utilized to make a single osteotomy. This is done by aligning the saw blade parallel to the k-wire. Care is taken to ensure that the lateral cortex is left intact. The medial wedge is created. This is done by inserting a series of three osteotomes. (Fig. 7) The $12 \mathrm{~mm}$ osteotome is inserted first, followed by the $10 \mathrm{~mm}$ osteotome, and lastly the $5 \mathrm{~mm}$ osteotome. All three osteotomes are then removed. 


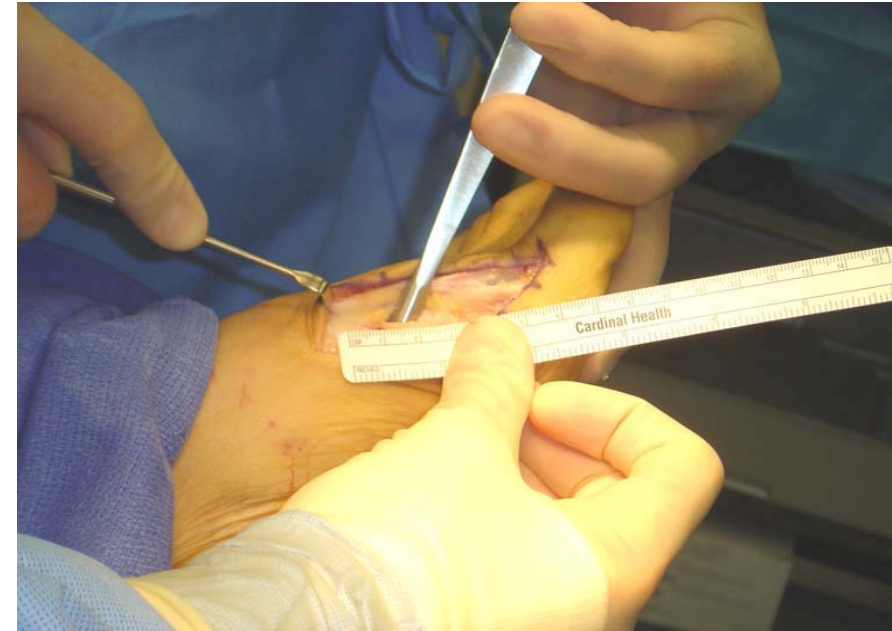

Figure 4 Measuring $1-1.5 \mathrm{~cm}$ distal to the metatarsalcuneiform joint for placement of the osteotomy.

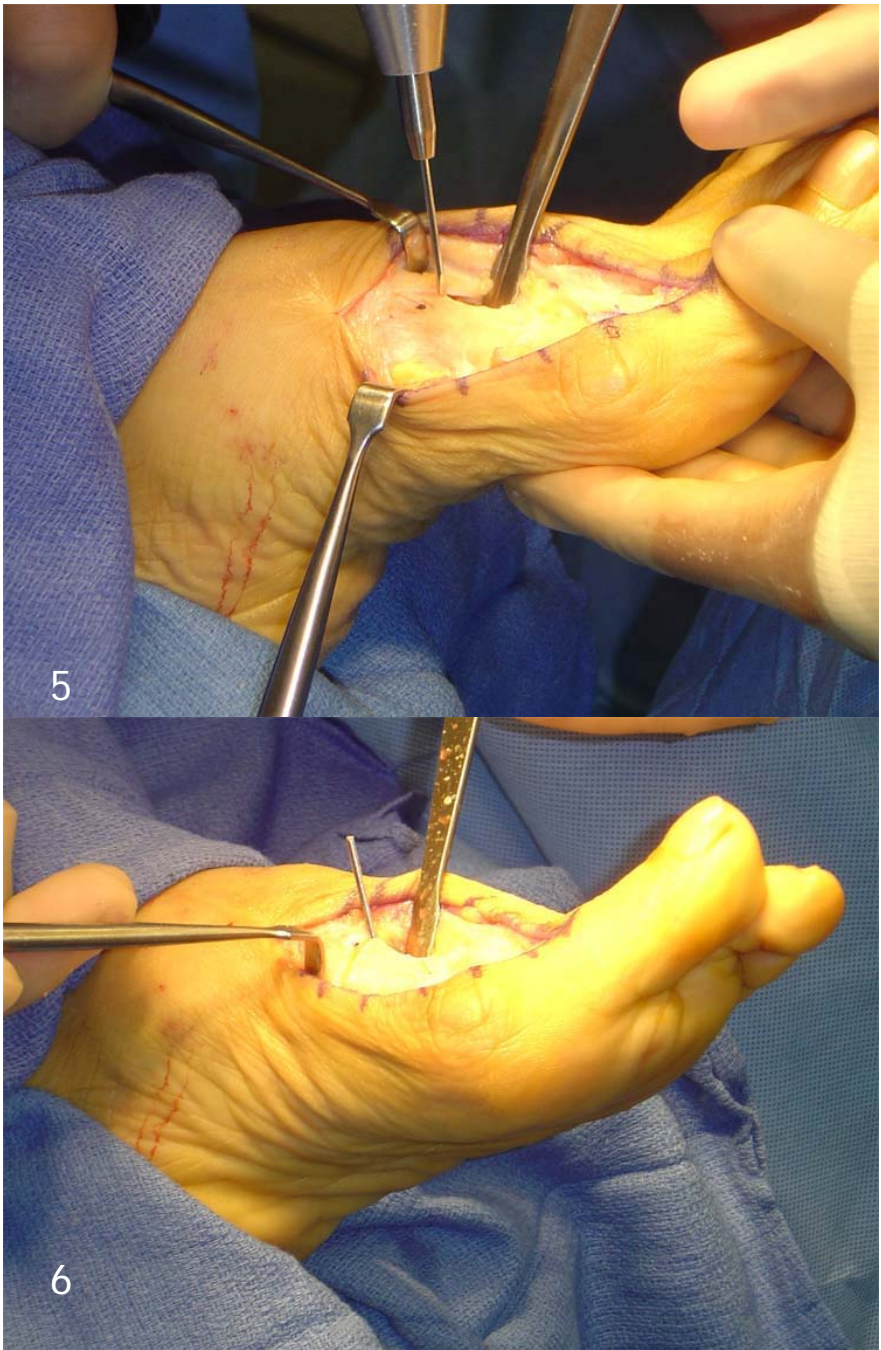

Figures 5 and 6 Placement of the axis guide wire perpendicular to the weight-bearing surface.

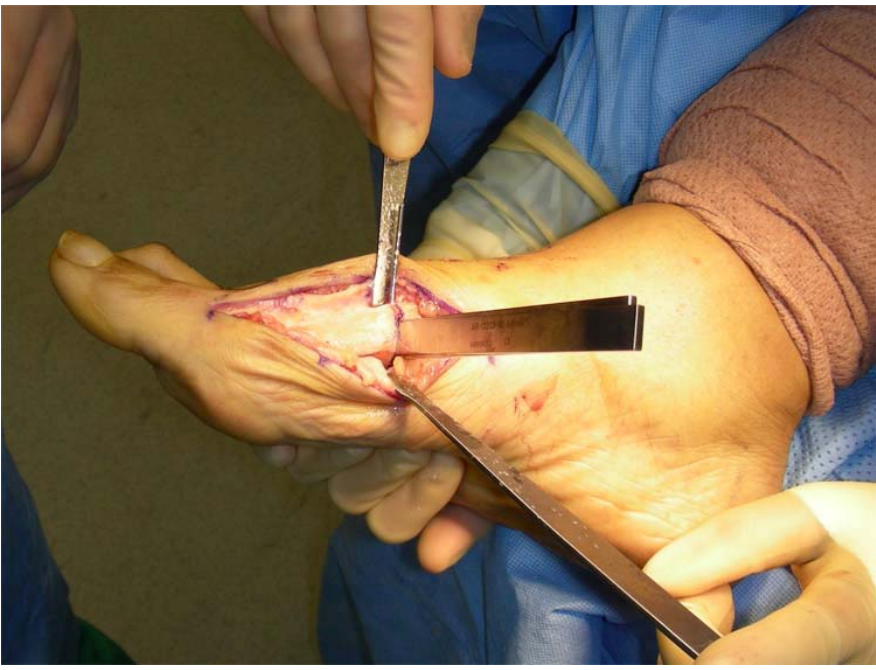

Figure 7 Multiple placement of osteotomes to incrementally distract the osteotomy. Care is taken to not break the lateral cortical hinge.

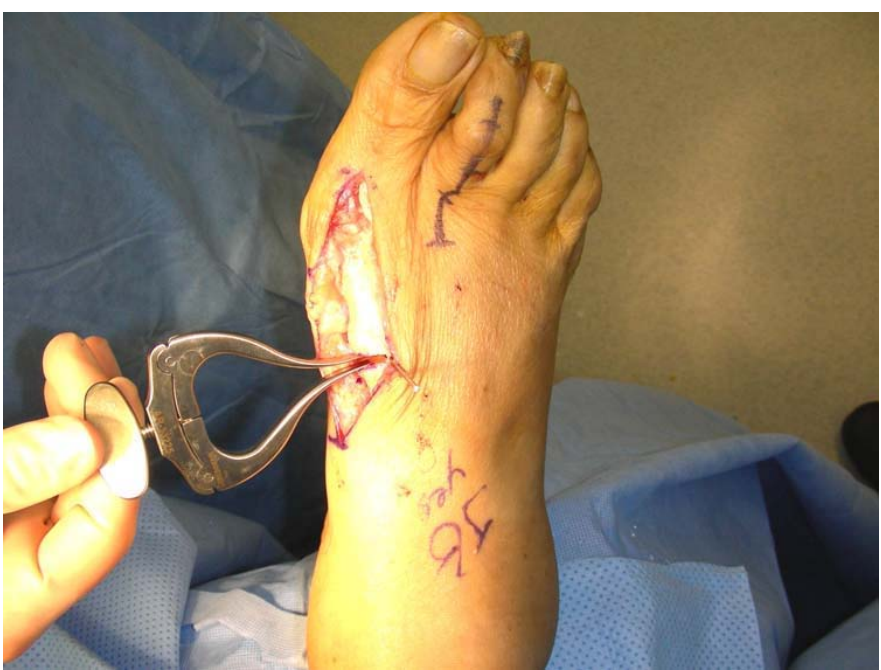

Figure 8 Demonstration of the Arthrex Osteotomy spreader. This is used to "dial in" the desired correction.

The osteotomy distractor is then placed medially and the wedge is opened to the desired width. (Fig. 8) Fluoroscopy is used to ensure that the intermetatarsal angle is adequately corrected.

The plate may then be placed directly on the medial surface of the metatarsal with the spacer flush against the proximal and distal edges of the wedge. The plate should be oriented such that the L-shaped portion is proximal to the osteotomy.

(C) The Foot and Ankle Online Journal, 2009 


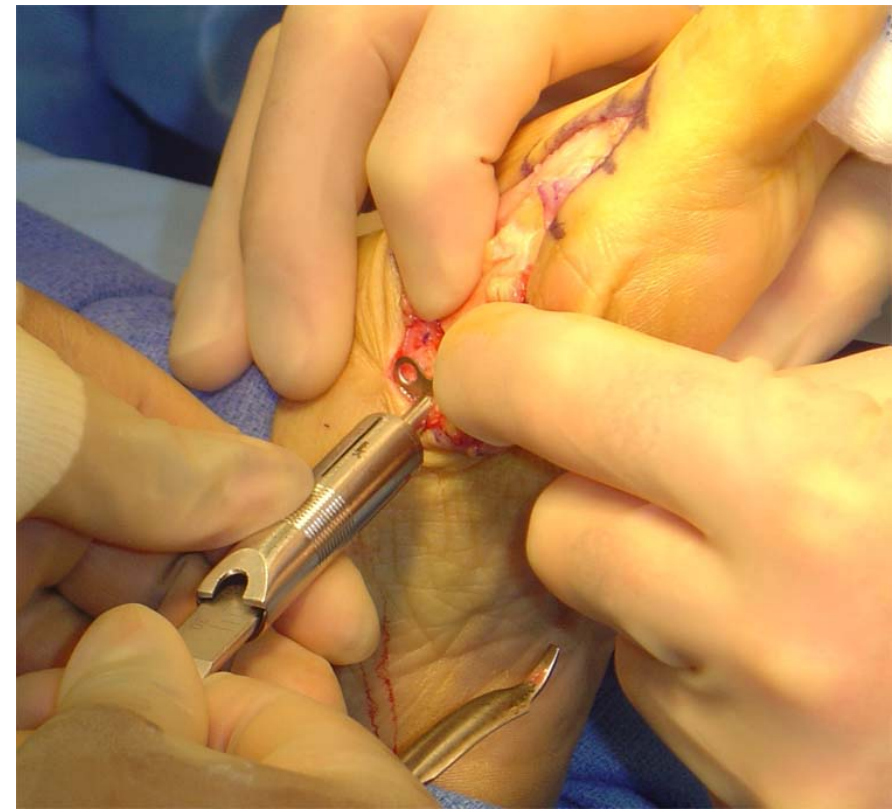

Figure 9 Placement of the low profile open wedge plate and measurement of appropriate screw length with the depth gauge.

The screws can then be used to fixate the opening wedge plate to the bone. The screws closest to the osteotomy should be inserted first. A $1.7 \mathrm{~mm}$ drill is passed through both cortices and the hole is measured with a depth gauge. The $2.3 \mathrm{~mm}$ fully threaded cortical screw is then inserted. (Fig. 9)

Once all screws are placed and the plate secured, the remaining void of the osteotomy should be filled with bone graft. This is usually done with a combination of the bone graft harvested from the medial eminence of the metatarsal head as well as allogenic cancellous bone chips. (Fig. 10 and 11) In hallux abduco valgus with larger intermetatarsal angles, there is a greater chance for compromise of the lateral cortical hinge. Should this occur, one can place an oblique screw across the osteotomy or even use a static plate from the Arthrex set. This can be applied dorsally along the first metatarsal to help prevent secondary elevatus. (Fig. 12, 13 and14)

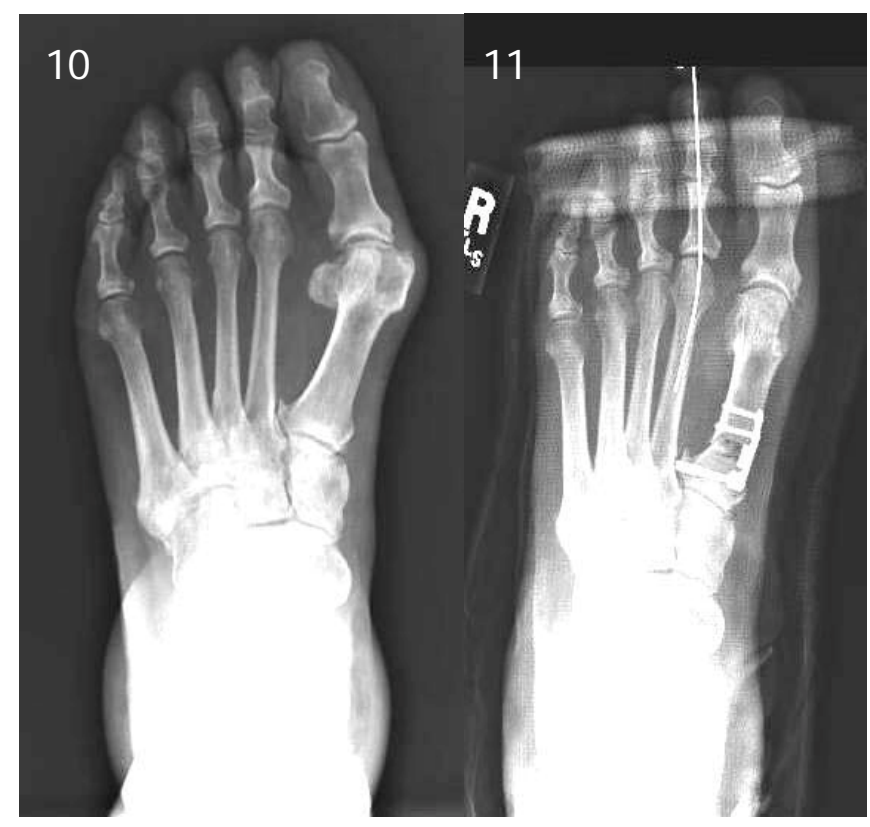

Figures 10 and 11 Pre and post operative films demonstrating the use of the Arthrex Low Profile Plate and Screw ${ }^{\mathrm{TM}}$.

\section{Discussion}

The opening base wedge technique was first described by Trethowin in 1923. ${ }^{1}$ In 1983, Beronio described using the medial eminence of the first metatarsal as a graft for the opening wedge osteotomy. ${ }^{2}$ Sollitto, et al., described an opening base wedge osteotomy with implant arthroplasty of the first metatarsophalangeal joint. They utilized the resected metatarsal head as a wedge graft. ${ }^{3}$ In the retrospective study of 20 patients, the average intermetatarsal angle was reduced from 16.13 to 3 degrees six months postoperatively. Limbirdm, et al., in 1989, reported results after performing an opening base wedge osteotomy and modified McBride bunionectomy in 15 patients. The intermetatarsal angle averaged 15 degrees preoperatively and 8 degrees postoperatively. ${ }^{4}$

The indications for an opening base wedge osteotomy include a short first metatarsal, intermetatarsal angle of 15 degrees or greater, adequate bone stock, and a compliant patient who understands the need for nonweight bearing. ${ }^{3}$ 


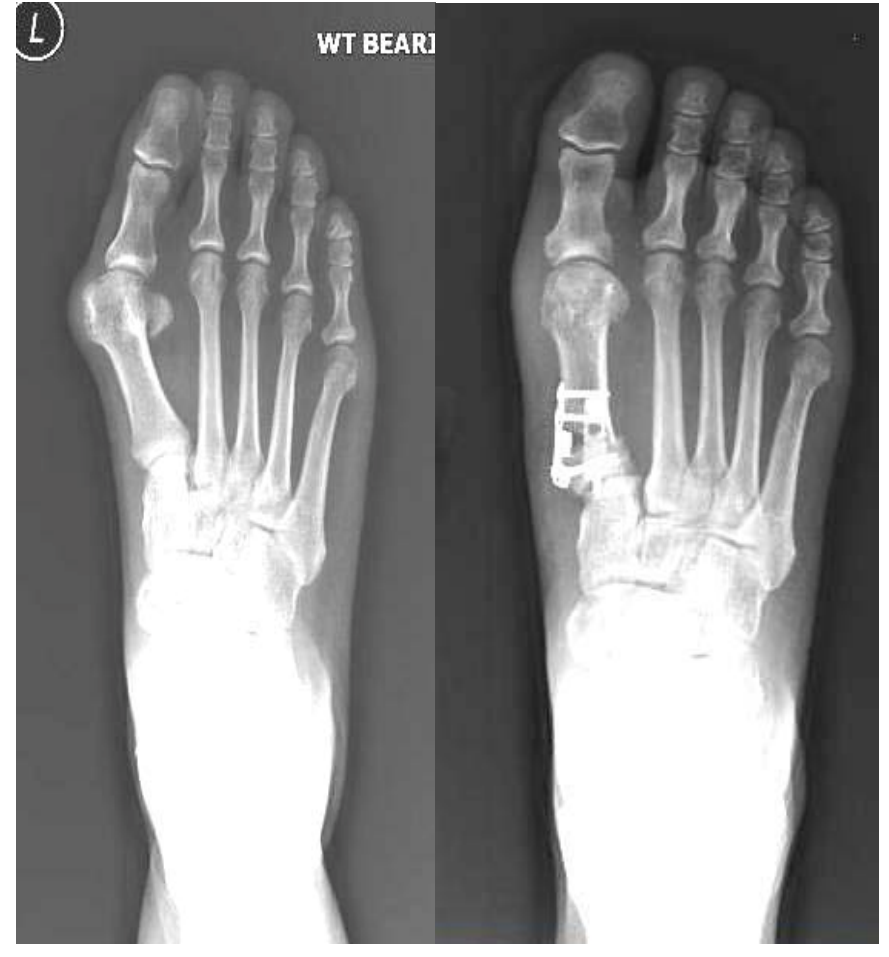

Figures 12 and 13 Use of an additional dorsal plate after compromise of the lateral cortex. For larger hallux valgus deformities, this helps prevent secondary postoperative elevatus.

Fixation for the opening base wedge osteotomy has varied. Trethowin felt that an intact lateral cortex and a properly fitting wedge graft were adequate and no further fixation was required. Other authors have agreed that no internal fixation was needed. ${ }^{3,4}$ Varied forms of internal fixation include screws, staples, cerclage wire, plates, and crossing pins. Amarnek, et al., described the use of the Hoffman miniature external fixator for immobilization of the first metatarsal osteotomy after bunionectomy. ${ }^{5}$

The opening base wedge osteotomy has had its critics. Many surgeons have felt that the opening wedge is difficult to perform and prefer to use other base wedge procedures. In the past, a bone graft had to be harvested and accurately sized in order to maintain proper correction of the metatarsal. The bone graft must be incorporated resulting in prolonged healing times. Scranton and Zuckerman noted that there was a rapid recurrence of the hallux abducto valgus deformity in 3 of 5 patients. ${ }^{6}$

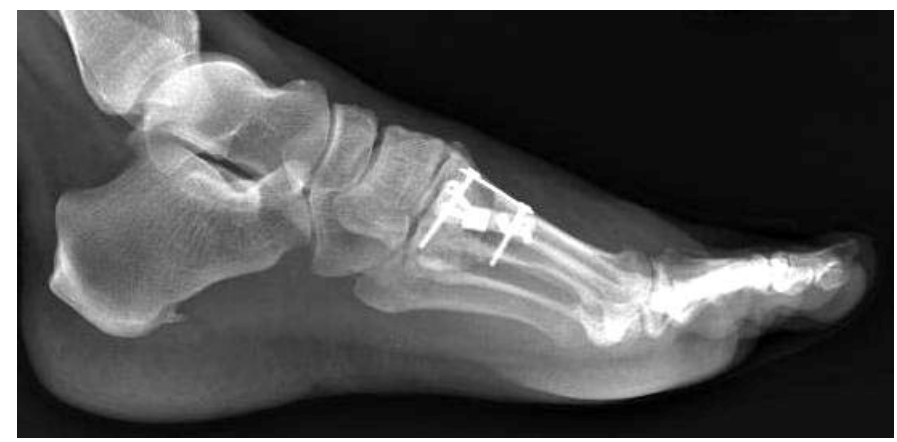

Figure 14 Lateral view showing the dorsal metatarsal plate in place.

They argued that the lengthening created with the opening wedge results in tightening of the soft tissue about the metatarsophalangeal joint, "promoting early hallux valgus recurrence." The lengthening of the metatarsal has also been thought to predispose the metatarsophalangeal joint to jamming and subsequent arthritis. $^{7}$ Other authors have also noted that the quality of the bone graft collected from the medial metatarsal head is suboptimal for incorporation.

With the development of the low profile plate and screw system by Arthrex, Inc., many of the aforementioned pitfalls can be prevented. The spacer on the plate acts as a pillar holding the wedge at the desired width. The Arthrex system eliminates the need for the bone graft to act as scaffolding for new bone growth. Moreover, it is not imperative that the surgeon make a precise fitting wedge of bone, allowing for the use of cancellous bone chips that will be more likely to incorporate into the bone. The plate is a permanent fixation device and will eliminate the risk of bunion recurrence.

\section{About the Low Profile Plate and Screw System}

The plates are titanium and have a thickness of $0.5 \mathrm{~mm}$. The plate spacers range from $2 \mathrm{~mm}$ to $7 \mathrm{~mm}$ in $0.5 \mathrm{~mm}$ increments. The titanium screws are $2.3 \mathrm{~mm}$ in diameter with a length ranging from $10 \mathrm{~mm}$ to $30 \mathrm{~mm}$ in $1 \mathrm{~mm}$ increments. The screws are fully threaded, self-tapping cortical design. The thread hole drill bit is $1.7 \mathrm{~mm}$. 


\section{Summary}

It is hoped that the Arthrex Low Profile Screw and Plate System ${ }^{\mathrm{TM}}$ will stimulate renewed interest of the opening base wedge osteotomy as a viable option for surgical correction of the hallux abducto valgus deformity. Many of the difficulties and complications seen in the past may be alleviated with this new design. The procedure is simple, offers adequate fixation of the osteotomy site, and ensures permanent correction.

\section{References}

1. Trethowan J. "Hallux valgus." In: Choyce CC (Ed), A System of Surgery. New York, P.B. Hoeber, 1046 - 1049, 1923.

2. Beronio JP. One approach to a viable method of obtaining cancellous bone for grafting. J Foot Surg, 22: 240 - 242, 1983.

3. Sollitto RJ, Hart TJ, Sergi AR. Opening base wedge osteotomy with first metatarsophalangeal joint implantation arthroplasty: A retrospective study. J Foot Surg: 30 (2) 165 - 169, 1991.

4. Limbird TJ, DaSilva RM, Green NE. Osteotomy of the first metatarsal base for metatarsus primus varus. Foot $\&$ Ankle. 9 (4): $158-162,1989$.

5. Amarnek DL, Juda EJ. Opening base wedge osteotomy of the first metatarsal utilizing rigid external fixation. J Foot Surg 25(4): $321-326,1986$.

6. Scranton PE, Zuckerman JD: Bunion surgery in adolescents: results of surgical treatment. J Pediatr Orthopaed 4 (1): 39 - 43, 1984.

7. Mothershed RA. Osteotomies of the first metatarsal base. In: Banks AS, Downey MS, Martin DE, Miller SJ (Eds) McGlamry's Comprehensive Textbook of Foot and Ankle Surgery. Philadelphia, Lippincott Williams \& Wilkins , 2001. 